\title{
Antiviral activity of sertindole, raloxifene and ibutamoren against transcription and replication-competent Ebola virus-like particles
}

\author{
Yi-Seul Yoon ${ }^{1,+}$, Yejin Jang ${ }^{1}$, Thomas Hoenen ${ }^{2}$, Heegwon Shin ${ }^{3}$, Younghoon Lee ${ }^{3}$ E Meehyein Kim ${ }^{1, *}$ \\ ${ }^{1}$ Virus Research Group, Korea Research Institute of Chemical Technology (KRICT), Daejeon 34114, Korea, ${ }^{2}$ Institute of Molecular Virology \\ and Cell Biology, Friedrich-Loeffler-Institut, Greifswald-Insel Riems 17493, Germany, ${ }^{3}$ Department of Chemistry, Korea Advanced \\ Institute of Science and Technology (KAIST), Daejeon 34141, Korea
}

\begin{abstract}
A chemical library comprising 2,354 drug-like compounds was screened using a transcription and replication-competent viruslike particle (trVLP) system implementing the whole Ebola virus (EBOV) life cycle. Dose-dependent inhibition of Ebola trVLP replication was induced by 15 hit compounds, which primarily target different types of $G$ protein-coupled receptors (GPCRs). Based on the chemical structure, the compounds were divided into three groups, diphenylmethane derivatives, promazine derivatives and chemicals with no conserved skeletons. The third group included sertindole, raloxifene, and ibutamoren showing prominent antiviral effects in cells. They downregulated the expression of viral proteins, including the VP40 matrix protein and the envelope glycoprotein. They also reduced the amount of EBOV-derived tetracistronic minigenome RNA incorporated into progeny trVLPs in the culture supematant. Particularly, ibutamoren, which is a known agonist of growth hormone secretagogue receptor (GHSR), showed the most promising antiviral activity with a $50 \%$ effective concentration of $0.2 \mu \mathrm{M}$, a $50 \%$ cytotoxic concentration of 42.4 $\mu \mathrm{M}$, and a selectivity index of 222.8. Here, we suggest a strategy for development of anti-EBOV therapeutics by adopting GHSR agonists as hit compounds. [BMB Reports 2020; 53(3): 166-171]
\end{abstract}

*Corresponding author. Tel: +82-42-860-7540; Fax: +82-42-8607400; E-mail: mkim@krict.re.kr

${ }^{\dagger}$ Present address: Center for Alternatives to Animal Testing, Korea Testing and Research Institute, Hwasun 58141, Korea

https://doi.org/10.5483/BMBRep.2020.53.3.175

Received 12 July 2019, Revised 20 August 2019, Accepted 16 October 2019

Keywords: Antiviral, Ebola virus, Ibutamoren, Raloxifene, Sertindole

\section{INTRODUCTION}

The genus Ebolavirus belonging to the family Filoviridae comprises five different species: Zaire, Sudan, Bundibugyo, Reston and Tai Forest (1). Zaire ebolavirus [ZEBOV or simply Ebola virus $(E B O V)]$ is an enveloped virus with a single-stranded, negative sense RNA genome measuring approximately 19,000 nucleotides in length (2). It encodes structural proteins including nucleoprotein (NP), envelope glycoprotein (GP), and the matrix protein VP40, in addition to VP24 (nucleocapsidassociated protein), VP30 (an activator of viral transcription), VP35 (a type I interferon antagonist and a polymerase cofactor) and RNA dependent RNA polymerase (L), and non-structural proteins such as sGP (3-6). Efficient viral infection requires at least two cellular receptors, the endo/lysosomal cholesterol transporter protein Niemann-Pick C1 (NPC1) and T-cell immunoglobulin and mucin domain 1 (TIM-1) $(7,8)$. The viral ribonucleocapsid (NC) consisting of the helical NP-viral RNA (vRNA) complex associated with VP24, VP30, VP35 and L is released into the cytoplasm after macropinocytosis (9). The negative sense VRNA is transcribed into mRNA for synthesis of viral proteins and complementary RNA for further vRNA amplification. During the final stage of the virus life cycle, the progeny virions are assembled at the plasma membrane via a VP40-dependent mechanism (10).

Since 1976 when this virus was first identified, its infection has caused Ebola hemorrhagic fever (EHF) in humans with average fatality rates exceeding $50 \%$ (available online at https:// www.who.int/news-room/fact-sheets/detail/ebola-virus-disease). The West African EBOV epidemic from 2014 to 2016 was the worst recorded EHF outbreak in history, resulting in 28,639 suspected and laboratory-confirmed cases and 11,316 deaths (available online at https://www.cdc.gov/vhf/ebola/history/20142016-outbreak/cost-of-ebola.html). More recently, the Zaire Ebola outbreak in the Democratic Republic of Congo caused at least 139 deaths as of August 2018 (11). Despite the high fatality rate, specific antiviral therapies are unavailable. Given the sporadic circulation of EBOV in Africa as well as the increased possibility of its international spread, it is imperative

ISSN: 1976-670X (electronic edition)

Copyright (c) 2020 by the The Korean Society for Biochemistry and Molecular Biology

(c) This is an open-access article distributed under the terms of the Creative Commons Attribution Non-Commercial License (http://creativecommons.org/licenses/by-nc/4.0) which permits unrestricted non-commercial use, distribution, and reproduction in any medium, provided the original work is properly cited. 
to develop prophylactic or therapeutic antivirals against EBOV infection (12).

Several high-throughput screening (HTS) methods, including monocistronic minigenome technology and pseudotyped viral particle systems, have been developed to identify antiviral compounds against EBOV (13-16). These strategies facilitated safe and robust screening of antivirals in biosafety level 2 (BSL2) facilities without the need for the highest-level bio-containment laboratory, BSL4, in which research with live EBOV is restricted. However, these methods enable limited targeting of the viral RNA transcription/replication or entry steps by the antiviral compounds. By contrast, the transcription and replication-competent virus-like particle (trVLP) system can be used to identify antiviral compounds by targeting virtually every stage of the viral life cycle (17). Here, the tetracistronic minigenomes carrying a reporter gene and three viral genes encoding structural proteins VP40, GP and VP24, flanked by 3'-leader and $5^{\prime}$-trailer regions, are cloned under the T7 promoter in the negative-sense orientation. Co-transfection of this tetracistronic construct with five additional plasmids expressing viral proteins for RNA synthesis, including NP, VP30, VP35 and L, together with T7 RNA polymerase initiates transcription and replication of the minigenome and leads to generation of infectious progeny VLPs encapsulating the minigenome RNA. Eventually, trVLPs acquire the ability to infect other target cells expressing TIM-1 and the four viral proteins, NP, VP30, VP35 and $\mathrm{L}$, for the second-round trVLP production. The aim of this study was to identify anti-EBOV compounds in a chemical library and to evaluate their antiviral efficacy using the trVLP system. Elucidation of the chemical structures of the resulting active compounds and the specific cellular targets provides scientific insight into the design of anti-EBOV drugs as well as their specific modes of action.

\section{RESULTS}

\section{Screening of a chemical library}

For high-throughput screening, we transfected and infected cells with the trVLP system derived from EBOV. After treatment of 2,354 small organic compounds, we measured their effects on the expression of both firefly luciferase (FLuc) and Renilla luciferase (RLuc), in which FLuc represents cell viability and RLuc denotes trVLP replication (Fig. 1). Based on the measurement of FLuc with $0.02 \%$ DMSO as a delivery vehicle, the average $Z^{\prime}$ value was 0.61 , with a signal-to-basal $(\mathrm{S} / \mathrm{B})$ ratio of 336 and a coefficient of variation $(\mathrm{CV})$ of $13 \%$ (Fig. 1B). From the RLuc analysis with $0.02 \%$ DMSO, the average $Z^{\prime}$ value was 0.57 , with an $S / B$ ratio of 450 and a CV of $14 \%$. $Z^{\prime}$ values in all plates were higher than 0.5 , which suggested the reliability of the screening assay. We primarily selected 86 hits with relative RLuc values $<15 \%$ and FLuc values ranging between $80 \%$ and $120 \%$ at $10 \mu \mathrm{M}$ (Fig. 1A, C).

\section{Evaluation of antiviral and cytotoxic effects}

Five-point dose-response curves with these 86 hits were analyzed with 3-fold dilutions from the initial concentration of $30 \mu \mathrm{M}$. Both $50 \%$ effective concentration $\left(\mathrm{EC}_{50}\right)$ and $50 \%$ cytotoxic concentration $\left(\mathrm{CC}_{50}\right)$ were calculated according to the simple two-point method using the RLuc and FLuc data, respectively (18). Fifteen compounds showed antiviral activity with selectivity indices (SI) exceeding 10 (Table 1). The majority of these compounds have been used to treat psychotic disorders or allergic diseases by binding to G protein-coupled receptors (GPCRs). This finding was partially consistent with previous reports of GPCR modulators as anti-EBOV compounds (19-21). Particularly, sertindole ( $\mathrm{EC}_{50}, 0.7 \mu \mathrm{M} ; \mathrm{SI},>45.8$ ), raloxifene hydrochloride (raloxifene; $\mathrm{EC}_{50}, 0.5 \mu \mathrm{M}$; SI, 47.1) and ibutamoren mesylate (ibutamoren; $\mathrm{EC}_{50},<0.4 \mu \mathrm{M} ; \mathrm{SI},>$ 81.1) showed the most prominent antiviral activity among the 15 major hit compounds. They were comparable or superior to the known anti-EBOV compounds such as ribavirin (RBV) and T-705 in their inhibitory effects.

To establish this finding, we purchased highly purified sertindole $(\geq 97.5 \%)$, raloxifene $(\geq 98 \%)$, and ibutamoren $(\geq$ $98 \%)$ and repeated the antiviral assay using a wide range of concentrations of compounds ( 0.015 to $100 \mu \mathrm{M}$ ) (Fig. 2). Nonlinear regression analysis revealed $\mathrm{EC}_{50}$ values of $1.5,1.2$, and $0.2 \mu \mathrm{M}$ and the $\mathrm{CC}_{50}$ values of $23.1,13.9$, and $42.4 \mu \mathrm{M}$ against HEK293T cells, respectively. However, the FLuc values
A

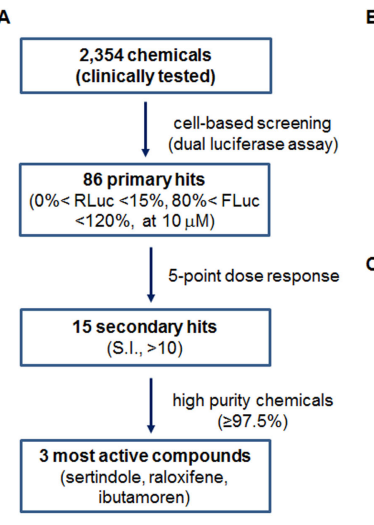

B

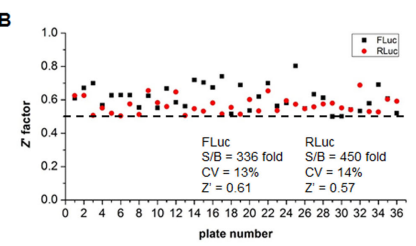

c

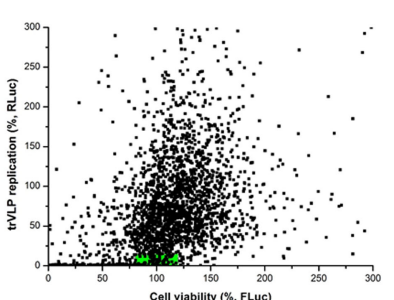

Fig. 1. Screening of anti-EBOV compounds using the trVLP system. (A) Flow chart showing the screening procedure. (B) $Z^{\prime}$ factor plot derived from HTS performed in 96-well plates. Black spots present the results of the FLuc assay and red spots those of the RLuc assay. The signal-to-basal ratio (S/B), coefficient of variation (CV), and average $Z^{\prime}$ factor are shown. The dashed line indicates a threshold of 0.5 . (C) trVLP replication (y-axis) and cell viability (x-axis) are expressed as percentages in the presence of 2,354 different compounds (each at $10 \mu \mathrm{M})$. The green box highlights 86 primary hits that suppressed trVLP replication below $15 \%$ and maintained viability of HEK293T cells between $80 \%$ and $120 \%$. 
Table 1. Anti-EBOV activity and cytotoxicity of the 15 hit compounds screened by dual luciferase assay using the trVLP system

\begin{tabular}{rlcccl}
\hline Hit no. & \multicolumn{1}{c}{ Compound } & $\mathrm{EC}_{50}{ }^{\mathrm{a}}(\mu \mathrm{M})$ & $\mathrm{CC}_{50}{ }^{\mathrm{b}}(\mu \mathrm{M})$ & $\mathrm{Sl}^{\mathrm{c}}$ & \\
\hline 1 & Homochlorcyclizine & $0.8 \pm 0.0$ & $>30.0$ & $>37.1$ & Mode-of-action \\
2 & Loperamide & $2.0 \pm 1.3$ & $>30.0$ & $>18.6$ & Opistamine H1-receptor antagonist \\
3 & Maprotiline & $1.2 \pm 0.7$ & $25.1 \pm 2.1$ & $26.9 \pm 18.1$ & Histamine H2 receptor inhibitor \\
4 & Benztropine mesylate & $3.1 \pm 1.4$ & $>30.0$ & $>10.9$ & Muscarinic acetylcholine M1 receptor antagonist \\
5 & Sertindole & $0.7 \pm 0.4$ & $>30.0$ & $>45.8$ & Dopamine D2 receptor inhibitor, \\
6 & 3-CPMT & serotonine 5-HT receptor inverse agonist \\
7 & Chlorcyclizine & $1.3 \pm 0.5$ & $>30.0$ & $>26.3$ & Dopamine transporter inhibitor \\
8 & Clemastine fumarate & $2.7 \pm 2.2$ & $>30.0$ & $>17.0$ & Histamine H1 receptor antagonist \\
9 & Triflupromazine & $1.0 \pm 0.5$ & $>30.0$ & $>10.2$ & Histamine H1 receptor antagonist \\
10 & Raloxifene & $0.5 \pm 0.0$ & $25.6 \pm 0.2$ & $47.1 \pm 2.2$ & Selective estrogen receptor modulator \\
11 & Proadifen & $2.7 \pm 0.7$ & $>30.0$ & $>11.6$ & Cytochrome P450 inhibitor, Ca antagonist \\
12 & Perphenazine & $0.7 \pm 0.2$ & $17.4 \pm 0.6$ & $27.7 \pm 9.2$ & Postsynaptic dopamine 2 receptor inhibitor \\
13 & lbutamoren mesylate & $<0.4$ & $>30.0$ & $>81.1$ & Growth hormone secretagogue agonist \\
14 & Piperacetazine & $2.0 \pm 0.6$ & $26.1 \pm 5.5$ & $15.7 \pm 4.6$ & Dopamine receptor antagonist \\
15 & SU11274 & $2.6 \pm 1.4$ & $>30.0$ & $>13.6$ & c-Met inhibitor \\
& RBV & $4.1 \pm 2.1$ & $>90.0$ & $>16.3$ & \\
& T-705 & $4.7 \pm 3.1$ & $>90.0$ & $>13.0$ & \\
\hline
\end{tabular}

${ }^{a}$ Half-maximal effective concentration estimated by the two-point method; ${ }^{b}$ half-maximal cytotoxic concentration by the two-point method; ${ }^{c}$ selectivity index, the ratio of $\mathrm{CC}_{50}$ to $\mathrm{EC}_{50} ;{ }^{\mathrm{d}} 3 \mathrm{a}$-[(4-chlorophenyl)phenylmethoxy]tropane.

could have been affected by the transfection efficiency of the reporter plasmid rather than by cell viability. Thus, we performed another cytotoxicity assay using 3-(4,5-dimethylthiazol-2-yl)-2,5-diphenyltetrazolium bromide (MTT). Treatment of the mock cells with increasing concentrations of the compounds resulted in $\mathrm{CC}_{50}$ values of $13.7 \mu \mathrm{M}$ for sertindole, $23.4 \mu \mathrm{M}$ for raloxifene, and $76.7 \mu \mathrm{M}$ for ibutamoren (supplementary Fig. 1), comparable to those derived from the dual luciferase assay. These results indicated that subtoxic levels of the three compounds exhibit anti-EBOV activity.

\section{Inhibition of viral protein expression and generation of viral progeny}

To investigate whether the antiviral compounds reduce viral protein levels and generation of progeny trVLP, we performed immunoassays and quantitative RT-PCR (Fig. 3). Western blot assay showed that tetracistronic minigenome-derived VP40 was downregulated in a dose-dependent manner by sertindole, raloxifene and ibutamoren as well as RBV, a positive control, whereas the amount of another viral protein, NP, expressed via the plasmid-derived CAG promoter remained consistent (Fig. 3A). Confocal laser scanning microscopy also confirmed the reduction of cytoplasmic GP levels in the trVLP-infected cells (Fig. 3B). Total fluorescent spots per well were quantified by collecting images from 36 areas without any overlap in each sample triplicate. This wide-field imaging analysis revealed that the number of GP-positive spots was reduced significantly in the presence of $10 \mu \mathrm{M}$ sertindole (by $84.7 \%$ ), raloxifene (by
$69.1 \%$ ), or ibutamoren (by $74.2 \%$ ) as well as $100 \mu \mathrm{M} \mathrm{RBV} \mathrm{(by}$ $62.5 \%$ ) (Supplementary Fig. 2). We investigated whether their inhibition suppressed the production of infectious trVLPs. Culture supernatants were harvested to quantify the relative amounts of the encapsidated tetracistronic minigenomic RNA. After RT and touchdown PCR, quantitative PCR analysis was performed using EBOV-specific primer sets as described previously (22). The results revealed suppression of the amount of negative-sense minigenome RNA incorporated into trVLPs in a dose-dependent manner (Fig. 3C). These data strongly suggested that sertindole, raloxifene and ibutamoren not only prevent trVLP-derived EBOV viral replication in cells but also subsequently block the generation of trVLP progeny.

\section{DISCUSSION}

Here, we identified 15 anti-EBOV compounds with selectivity indices higher than 10 by screening a chemical library of compounds tested for clinical trials. Based on their target analysis, we found that GPCRs were closely linked to the EBOV life cycle. Using the same trVLP system, N. Lee et al. found GPCR modulators as antiviral hits, suggesting experimental reliability (19). However, in contrast to the previous study, from a technical perspective, we adopted the dual luciferase assay to measure the antiviral activity and cytotoxicity simultaneously from the same sample, while the cytotoxicity was determined in an image-based manner via Hoechst staining. Second, we evaluated the antiviral activity of the 
A

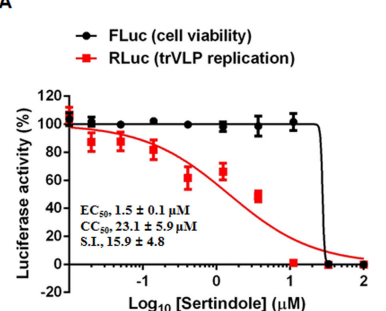

c

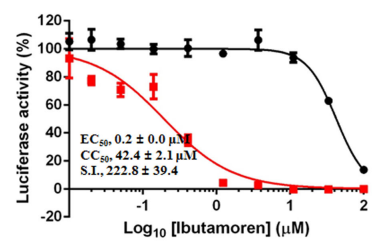

B

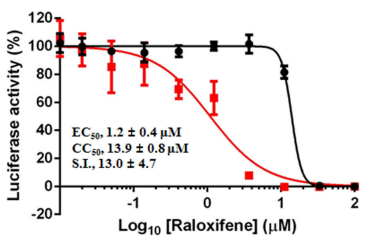

D

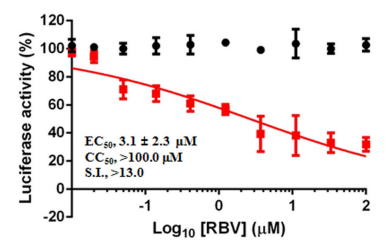

Fig. 2. Antiviral activity of highly purified sertindole, raloxifene, and ibutamoren. HEK293T cells were applied to the trVLP system in the presence of increasing concentrations of sertindole (A), raloxifene (B) or ibutamoren (C) using RBV (D) as a control. Percentage cell viability (black circles) and percentage trVLP replication (red squares) were assessed in FLuc and RLuc assays, respectively. $\mathrm{EC}_{50}$ and $\mathrm{CC}_{50}$ values were calculated via non-linear regression analysis using the GraphPad Prism 6 software. The means \pm standard deviations of the $\mathrm{EC}_{50}, \mathrm{CC}_{50}$, and $\mathrm{SI}$ values are shown on the corresponding panels. These data are derived from two independent experiments in triplicate.

selected compounds by using immunoblotting or genetic analysis alternatively, independent of the luciferase activity. It is not trivial, because hit compounds targeting the reporter protein, either FLuc or RLuc, were selected accidentally and frequently (23). In addition, classification of the chemical structure facilitated the determination of structure-activity relationships (Supplementary Fig. 3). Diphenylmethane derivatives belong to group I, promazine derivatives to group II and other compounds to group III. Given that the histamine $\mathrm{H} 1$ receptor of GPCRs is rarely expressed in human embryonic kidney 293 (HEK293) cells (24), a parental cell line of HEK293T, and that groups I and II carry distinct chemical skeletons as diphenylmethane and promazine cores, respectively, these structural features might be preferred for the induction of antiviral activity. Furthermore, we tested the antiviral efficacy of the three active compounds including sertindole, raloxifene and ibutamoren against other human RNA viruses, such as dengue virus and rhinovirus (25-27). We found no viral inhibition in cells, indicating that the hit compounds are EBOV-specific (data not shown).

Among the selected hits, ibutamoren exhibited the most potent inhibitory effect against $\mathrm{EBOV}$, with an $\mathrm{EC}_{50}$ of $0.2 \mu \mathrm{M}$, a $\mathrm{CC}_{50}$ of $42.4 \mu \mathrm{M}$ and a SI of 222.8 (Fig. 2C). The compound (also called MK-677) is an orally active agonist binding to a

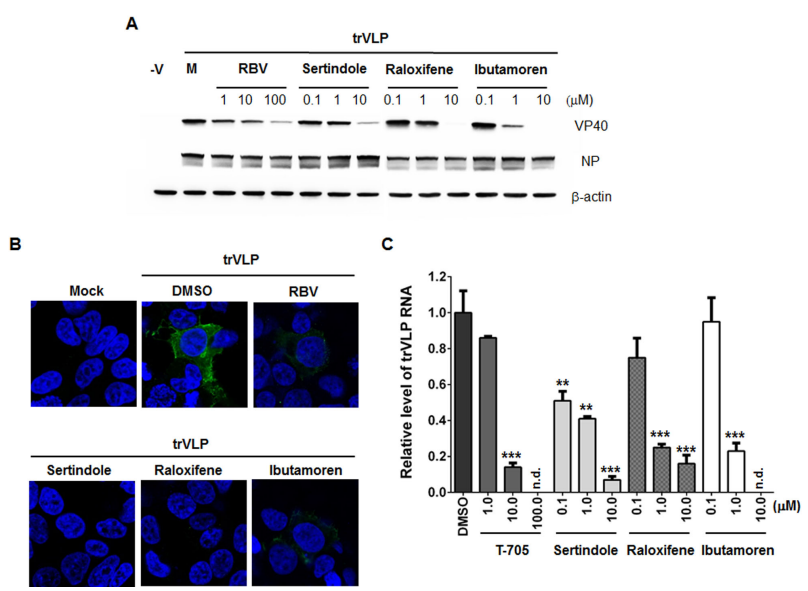

Fig. 3. Inhibition of viral protein expression and progeny trVLP generation. (A) Western blot analysis to detect VP40. Cells were transfected and infected with trVLP with increasing concentrations of each compound. On day 2 p.i., immunoblot analysis was performed with antibodies specific for VP40 (upper), NP (middle), or $\beta$-actin (lower). $-\mathrm{V}$, no transfection and no infection control. $M$, trVLP-infected, DMSO-treated mock control. (B) Confocal microscopy for detection of viral GP. HEK293T cells were transfected and infected with trVLP and then treated with DMSO $(0.2 \% \mathrm{v} / \mathrm{v})$, $100 \mu \mathrm{M}$ RBV, or $10 \mu \mathrm{M}$ of each hit compound (sertindole, raloxifene or ibutamoren). On day 1 p.i., cells were fixed and permeabilized to probe the viral GP with an anti-GP antibody and an Alexa488-conjugated secondary antibody (green). Nuclei were counterstained with DAPI (blue). Original magnification, $\times$ 400. (C) Quantitative RT-PCR for measurement of trVLP RNA levels in the culture supernatants. HEK293T cells transfected with the plasmids mentioned above and infected with trVLP were treated with increasing concentrations of T-705, sertindole, raloxifene, or ibutamoren. On day 2 p.i., the trVLP RNA was purified from the supernatants and subjected to real-time RT-PCR with EBOV GP-specific primer sets. The experiment was performed in triplicate. Statistical significance was estimated using the DMSOtreated sample as a control. n.d., not detected. $* * P<0.01$; $* * * \mathrm{P}<0.001$.

GPCR, the growth hormone secretagogue receptor (GHSR), that is a known ghrelin receptor. This receptor increases $\mathrm{GH}$ levels, fat-free mass, and energy expenditure; however, due to increased cortisol levels, it has yet to be approved by the US Food and Drug Administration (FDA) $(28,29)$. Nevertheless, the anti-EBOV activity mediated via GHSR inhibition has implications for target discovery, because GHSR acts as a hub in the GPCR network of intra- or inter-family connections (30). The mechanism underlying its antiviral activity as well as evaluation of its efficacy against wild-type, highly pathogenic Ebola viral strains remains to be investigated. In the absence of any clinically available antiviral drug, this study informs that stimulation of GHSR represents a strategic and efficient approach for the development of a host-targeted anti-EBOV drug. 


\section{MATERIALS AND METHODS}

\section{Cells, plasmids and chemicals}

HEK293T cells were purchased from the American Type Culture Collection (ATCC) and maintained in Dulbecco's Modified Eagle's Medium (DMEM; Hyclone) supplemented with $10 \%$ fetal bovine serum (FBS; Hyclone).

Plasmids used in this study were designed for the tetracistronic minigenome (p4cis-vRNA-RLuc) under the T7 promoter and for the expression of T7 RNA polymerase (pCAGGS-T7), viral proteins (pCAGGS-NP, -VP30, -VP35 and -L) and TIM-1 (pCAGGS-Tim1) under the chicken beta-actin (CAG) promoter. Their constructs were reported previously (17).

We obtained 2,354 chemicals either approved by or investigated in clinical trials undertaken by the FDA, the European Medicines Agency (EMA), or other agencies: 1,600 from the Pharmakon-1600 collection (MicroSource Discovery Systems), 326 from the Prestwick Chemical Library (Prestwick Chemical), 103 from the Tocriscreen Library (Tocris Bioscience), and 325 from an in-house library (the Korea Chemical Bank) deposited by domestic chemists. Highly purified sertindole $(\geq$ $97.5 \%)$ and ibutamoren $(\geq 98 \%)$ were purchased from Sigma-Aldrich, and raloxifene ( $\geq 98 \%$ ) from Santa Cruz Biotechnology. Control antiviral compounds, RBV and favipiravir (T-705; 6-flouro-3-hydroxy-2-pyrazinecarboxamide) were purchased from Sigma-Aldrich or chemically synthesized in-house (patent application no., EP1417967A4).

\section{trVLP generation and cell-based HTS}

HEK293T cells $\left(2 \times 10^{7}\right.$ cells per dish) were cultured in a $150-$ $\mathrm{mm}$ dish overnight and transfected with p4cis-vRNA-RLuc (4 $\mu \mathrm{g})$, pCAGGS-T7 $(4 \mu \mathrm{g})$, -NP $(2 \mu \mathrm{g})$, -VP35 $(2 \mu \mathrm{g})$, -VP30 $(1 \mu \mathrm{g})$, and L (15 $\mu \mathrm{g})$ using Lipofectamine 2000 (Invitrogen) according to the manufacturer's instructions. On day 3 post-transfection, the culture supernatants with EBOV-derived trVLPs (passage 0, P0) were harvested and centrifuged at 1,000 rpm for $5 \mathrm{~min}$ $(17,22)$. Their aliquots were stored at $-70^{\circ} \mathrm{C}$ before use.

HTS was performed in 96-well plates using the transfection/ infection method. Briefly, HEK293T cells $\left(3 \times 10^{4}\right.$ per well $)$ were cultured overnight and transfected with a plasmid set consisting of pCAGGS-NP (8 ng), -VP35 (8 ng), -VP30 (5 ng), -L (60 ng) together with pCAGGS-Tim1 (15 ng) as well as pEGFPLuc (0.1 ng; BD Bioscience Clontech), which contains Firefly luciferase (FLuc). The next day, cells were infected with trVLPs (P0) diluted 1:10 with culture medium in the presence of each test compound (at $10 \mu \mathrm{M}$ ). On day 2 post-infection (p.i.), the cell lysates were harvested to determine the antiviral activity and cytotoxicity by measuring RLuc and FLuc levels, respectively, using a dual luciferase assay (Promega).

\section{Western blot analysis}

HEK293T cells seeded in 6-well plates $\left(1 \times 10^{6}\right.$ cells per well) were transfected with pCAGGS-NP (120 ng), -VP35 (120 ng), -VP30 (80 ng), -L (1,000 ng) together with pCAGGS-Tim1 (250 ng). The next day, $2 \mathrm{~mL}$ of 3-fold diluted trVLPs (P0) were infected into cells with increasing concentrations of antiviral compounds. On day 1 p.i., the cell lysates were harvested using the M-PER buffer (Thermo Scientific). Twenty micrograms of total protein per well were loaded on the $10 \%$ or $12 \%$ SDS-PAGE and electrophoretically transferred to a polyvinylidene fluoride membrane (Millipore). Viral proteins were detected using primary antibodies specific for VP40 (Cat. no. 40446-T48; Sino Biological) or NP (Cat. no. 40443-T62; Sino Biological), followed by a horseradish peroxidase (HRP)-conjugated goat anti-rabbit secondary antibody (Thermo Scientific). The cellular $\beta$-actin protein, a loading control, was detected with an anti- $\beta$-actin-specific primary antibody (Cat. No. A1987; Sigma) and the HRP-conjugated goat anti-mouse secondary antibody (Thermo Scientific).

\section{Confocal microscopy}

Plasmids expressing polymerase complex (NP, VP35, VP30, and L) and TIM-1 were cotransfected into HEK293T cells seeded on 12-mm glass cover slips in 24-well plates $\left(3 \times 10^{5}\right.$ cells per well). The next day, the cells were infected with 500 $\mu \mathrm{l}$ of trVLP (P0) in the presence of each test compound (10 $\mu \mathrm{M})$ or RBV $(100 \mu \mathrm{M})$. Following fixation and permeabilization, the cells were immuno-stained with an anti-GP antibody (Cat. no. 40304-T44; Sino Biological) and an Alexa 488-conjugated goat anti-rabbit antibody (Invitrogen), and counterstained with Vectashield mounting medium containing 4,6-diamidino2-phenylindole (DAPI; Vector Laboratories). Confocal fluorescent images were acquired using Zeiss LSM 700 (Carl Zeiss). The number of GP-positive cells was quantified by collecting data from 36 nonoverlapping images per well in triplicate for each sample to analyze wide-field fluorescence occupancy (IncuCyte FLR; Essen BioScience).

\section{Real-time reverse transcription-polymerase chain reaction (RT-PCR)}

The level of tetracistronic minigenome RNA incorporated within trVLP (P1) was measured by harvesting the culture supernatants from the samples prepared according to a previous report (22). In brief, tetracistronic trVLP RNA was purified using a QIAamp Viral RNA kit (Qiagen) on day 2 p.i. Following reverse-transcription, cDNA was applied to a touchdown PCR with an EBOV-specific primer set followed by quantitative PCR with another inner primer set using SsoFast EvaGreen Supermix (Bio-Rad) and the CFX96 real-time PCR detection system (Bio-Rad). To calculate the relative fold changes, the RNA level in the DMSO-treated, trVLP-infected culture supernatant was set at 1 .

\section{ACKNOWLEDGEMENTS}

M. K. was supported by grants from KRICT (KK1803-E00 and KK2032-20). Y. L. was supported by the National Research Foundation of Korea (NRF) grant funded by the Korea govern- 
ment (MSIT) (No. NRF-2019R1H1A2039730). H. S was supported by the NRF grant funded by the Korea government (MSIT) (No. NRF-2017R1A6A3A11031308). We thank the Korea Chemical Bank for providing the chemicals for HTS.

\section{CONFLICTS OF INTEREST}

The authors have no conflicting interests.

\section{REFERENCES}

1. Kuhn JH, Becker S, Ebihara $\mathrm{H}$ et al (2010) Proposal for a revised taxonomy of the family Filoviridae: classification, names of taxa and viruses, and virus abbreviations. Arch Virol 155, 2083-2103

2. Jun SR, Leuze MR, Nookaew I et al (2015) Ebolavirus comparative genomics. FEMS Microbiol Rev 39, 764-778

3. Schumann M, Gantke T and Muhlberger E (2009) Ebola virus VP35 antagonizes PKR activity through its C-terminal interferon inhibitory domain. J Virol 83, 8993-8997

4. McCarthy SE, Johnson RF, Zhang YA, Sunyer JO and Harty RN (2007) Role for amino acids 212KLR214 of Ebola virus VP40 in assembly and budding. J Virol 81, 11452-11460

5. Biedenkopf N, Schlereth J, Grunweller A, Becker S and Hartmann RK (2016) RNA Binding of Ebola Virus VP30 Is Essential for Activating Viral Transcription. J Virol 90, 7481-7496

6. Banadyga L, Hoenen T, Ambroggio X, Dunham E, Groseth A and Ebihara H (2017) Ebola virus VP24 interacts with NP to facilitate nucleocapsid assembly and genome packaging. Sci Rep 7, 7698

7. Carette JE, Raaben M, Wong AC et al (2011) Ebola virus entry requires the cholesterol transporter Niemann-Pick C1. Nature 477, 340-343

8. Kondratowicz AS, Lennemann NJ, Sinn PL et al (2011) T-cell immunoglobulin and mucin domain 1 (TIM-1) is a receptor for zaire ebolavirus and lake victoria marburgvirus. Proc Natl Acad Sci U S A 108, 8426-8431

9. Sugita $Y$, Matsunami $H$, Kawaoka $Y$, Noda T and Wolf $M$ (2018) Cryo-EM structure of the Ebola virus nucleoproteinRNA complex at 3.6 A resolution. Nature 563, 137-140

10. Jasenosky LD, Neumann G, Lukashevich I and Kawaoka Y (2001) Ebola virus VP40-induced particle formation and association with the lipid bilayer. J Virol 75, 5205-5214

11. Arie S (2018) Ebola: WHO expresses "very serious concern" about Congo outbreak. BMJ 363, k4430

12. Cohen NJ, Brown CM, Alvarado-Ramy F et al (2016) Travel and border health measures to prevent the international spread of Ebola. MMWR Suppl 65, 57-67

13. Edwards MR, Pietzsch C, Vausselin T, Shaw ML, Bukreyev A and Basler CF (2015) High-Throughput Minigenome System for Identifying Small-Molecule Inhibitors of Ebola Virus Replication. ACS Infect Dis 1, 380-387

14. Kouznetsova J, Sun W, Martinez-Romero C et al (2014) Identification of 53 compounds that block Ebola virus-like particle entry via a repurposing screen of approved drugs. Emerg Microbes Infect 3, e84

15. Wang Y, Cui R, Li G et al (2016) Teicoplanin inhibits
Ebola pseudovirus infection in cell culture. Antiviral Res $125,1-7$

16. Welch SR, Guerrero LW, Chakrabarti AK et al (2016) Lassa and Ebola virus inhibitors identified using minigenome and recombinant virus reporter systems. Antiviral Res 136, 9-18

17. Hoenen T, Watt A, Mora A and Feldmann H (2014) Modeling the lifecycle of Ebola virus under biosafety level 2 conditions with virus-like particles containing tetracistronic minigenomes. J Vis Exp, 52381

18. Nevozhay D (2014) Cheburator software for automatically calculating drug inhibitory concentrations from in vitro screening assays. PLoS One 9, e106186

19. Lee N, Shum D, Konig A et al (2018) High-throughput drug screening using the Ebola virus transcription- and replication-competent virus-like particle system. Antiviral Res 158, 226-237

20. Fan H, Du X, Zhang J et al (2017) Selective inhibition of Ebola entry with selective estrogen receptor modulators by disrupting the endolysosomal calcium. Sci Rep 7, 41226

21. Cheng $\mathrm{H}$, Lear-Rooney $\mathrm{CM}$, Johansen $\mathrm{L}$ et al (2015) Inhibition of Ebola and Marburg Virus Entry by G ProteinCoupled Receptor Antagonists. J Virol 89, 9932-9938

22. Watt A, Moukambi F, Banadyga L et al (2014) A novel life cycle modeling system for Ebola virus shows a genome length-dependent role of VP24 in virus infectivity. J Virol 88, 10511-10524

23. Thorne N, Shen M, Lea WA et al (2012) Firefly luciferase in chemical biology: a compendium of inhibitors, mechanistic evaluation of chemotypes, and suggested use as a reporter. Chem Biol 19, 1060-1072

24. Atwood BK, Lopez J, Wager-Miller J, Mackie K and Straiker A (2011) Expression of $G$ protein-coupled receptors and related proteins in HEK293, AtT20, BV2, and N18 cell lines as revealed by microarray analysis. BMC Genomics 12,14

25. Jung E, Nam S, Oh H et al (2019) Neutralization of acidic intracellular vesicles by niclosamide inhibits multiple steps of the dengue virus life cycle in vitro. Sci Rep 9, 8682

26. Kim J, Shin JS, Ahn S, Han SB and Jung YS (2018) 3-Aryl-1,2,4-oxadiazole derivatives active against human rhinovirus. ACS Med Chem Lett 9, 667-672

27. Jang Y, Shin JS, Yoon YS et al (2018) Salinomycin inhibits influenza virus infection by disrupting endosomal acidification and viral matrix protein 2 function. J Virol 92, e01441-18

28. Svensson J, Lonn L, Jansson JO et al (1998) Two-month treatment of obese subjects with the oral growth hormone $(\mathrm{GH})$ secretagogue MK-677 increases $\mathrm{GH}$ secretion, fat-free mass, and energy expenditure. I Clin Endocrinol Metab 83, 362-369

29. Patchett AA, Nargund RP, Tata JR et al (1995) Design and biological activities of L-163,191 (MK-0677): a potent, orally active growth hormone secretagogue. Proc Natl Acad Sci U S A 92, 7001-7005

30. Borroto-Escuela DO, Brito I, Romero-Fernandez W et al (2014) The G protein-coupled receptor heterodimer network (GPCR-HetNet) and its hub components. Int J Mol Sci 15, 8570-8590 